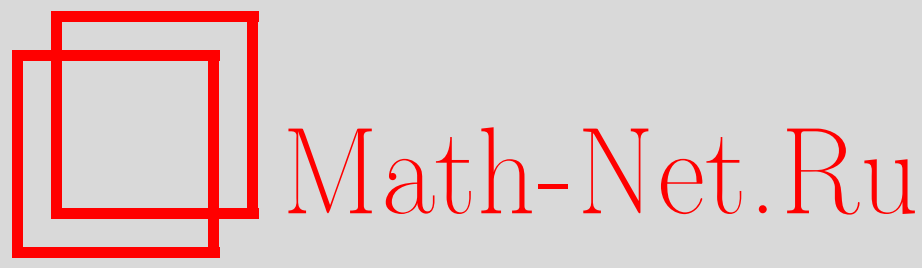

И. Я. Арефьева, Р. В. Горбачев, О калибровочной эквивалентности тахионных решений в кубической полевой теории струн Невё-Шварца, ТМФ, 2010, том 165, номер 2, 323-328

DOI: https://doi.org/10.4213/tmf6579

Использование Общероссийского математического портала Math-Net.Ru подразумевает, что вы прочитали и согласны с пользовательским соглашением http://www . mathnet.ru/rus/agreement

Параметры загрузки:

IP : 54.80 .97 .219

26 апреля 2023 г., 15:15:34

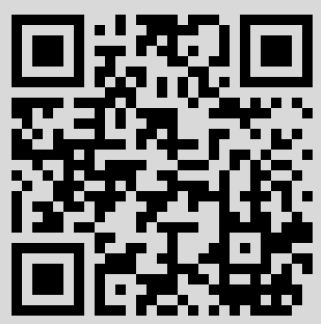




\section{О КАЛИБРОВОЧНОЙ ЭКВИВАЛЕНТНОСТИ ТАХИОННЫХ РЕШЕНИЙ В КУБИЧЕСКОЙ ПОЛЕВОЙ ТЕОРИИ СТРУН НЕВЁ-ШВАРЦА}

Построено простое аналитическое решение кубической полевой теории струн Невё-Шварца, включающей GSO(-)-сектор. Это решение аналогично решению Эрлера-Шнабла для бозонного случая и решению для чистого $\mathrm{GSO}(+)$-сектора, ранее предложенному одним из авторов. Построено точное калибровочное преобразование нового решения в другие известные решения для тахионной конденсации струны Невё-Шварца. Эта калибровочная эквивалентность подтверждает сделанное ранее наблюдение, что решение Эрлера для чистого $\mathrm{GSO}(+)$ сектора и наше решение, содержащее GSO(+)- и GSO(-)-секторы, имеют одинаковую величину плотности действия.

Ключевые слова: полевая теория струн, конденсация тахиона, D-браны.

\section{1. ВВЕДЕНИЕ}

Первое нетривиальное вакуумное решение уравнения движения полевой теории струн (ПТС) было найдено в основополагающей статье Шнабла [1]. Это решение может быть представлено как сингулярный предел чисто калибровочной конфигурации [1], [2]. В работе Шнабла было получено решение в виттеновской бозонной ПТС [3], а в работе [4] - подобное решение в кубической полевой теории суперструны [5], [6]. Решение в фермионной ПТС, включающей GSO(-)-сектор [7], было построено в работах [8], [9]. И решение Эрлера $\Phi_{\mathrm{E}}[4]$, и наше решение $\widehat{\Phi}_{\mathrm{AGM}}$ из работ [8], [9] (далее мы называем его решением АГМ) удовлетворяют первой и третьей гипотезам Сена. Так как оба эти решения являются сингулярным пределом чисто калибровочной конфигурации, можно полагать, что они связаны калибровочным преобразованием [10]. Для работы с построенными вакуумными решениями было бы неплохо построить сглаживающее калибровочное преобразование. Гладкое вакуумное решение бозонной ПТС было недавно найдено в работе [11]. Следуя терминологии этой работы, мы будем называть его простым аналитическим решением.

* Математический институт им. В. А. Стеклова РАН, Москва, Россия. E-mail: arefeva@mi.ras.ru,rgorbachev@mi.ras.ru 
Простое аналитическое решение уравнения движения кубической полевой теории суперструн построено в работе [12].

В настоящей статье мы строим простое аналитическое решение уравнений движения фермионной ПТС с ненулевым GSO(-)-сектором. Подобно случаям, рассмотренным в работах [11], [12], наше новое решение содержит интеграл от веджевских состояний и не использует сингулярного предела. Мы демонстрируем калибровочную эквивалентность этого решения и простого решения в $\mathrm{GSO}(+)$-секторе. Данный результат является ожидаемым, так как оба решения имеют одинаковую величину действия, однако он нетривиален, поскольку они не являются чистой калибровкой. Мы также приводим калибровочное преобразование, связывающее новое аналитическое решение с аналитическим решением АГМ из статьи [8]. Заметим, что калибровочное преобразование, связывающее чистое простое решение $\Phi_{\mathrm{G}}$ в $\mathrm{GSO}(+)$-секторе [12] и соответствующее решение с фантомным слагаемыми $\Phi_{\mathrm{E}}$ из работы [4], было приведено в работе [12].

Мы имеем следующую картину:

$$
\Phi_{\mathrm{E}} \stackrel{U_{\mathrm{E}, \mathrm{G}}}{\longrightarrow} \Phi_{\mathrm{G}}, \quad \widehat{\Phi}_{\mathrm{AGM}} \stackrel{\widehat{U}_{\mathrm{AGM}, \text { new }}}{\longrightarrow} \widehat{\Phi}_{\text {new }},
$$

где первое соотношение означает, что существует калибровочное преобразование $U_{\mathrm{E}, \mathrm{G}}$, переводящее решение $\Phi_{\mathrm{E}}$ в решение $\Phi_{\mathrm{G}}$, второе соотношение - что существует преобразование $\widehat{U}_{\mathrm{AGM}, \mathrm{new}}$, переводящее решение $\widehat{\Phi}_{\mathrm{AGM}}$ в новое решение $\widehat{\Phi}_{\text {new }}$, представленное в настоящей статье. Как было отмечено выше, мы можем построить калибровочное преобразование $\widehat{U}_{\mathrm{G}, \mathrm{new}}$, связывающее решения $\Phi_{\mathrm{G}}$ и $\widehat{\Phi}_{\text {new }}$; таким образом, мы можем замкнуть диаграмму:

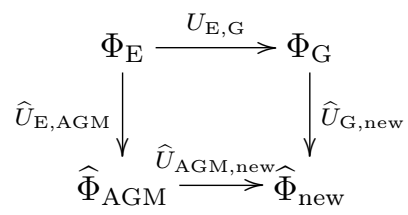

и

$$
U_{\mathrm{E}, \mathrm{G}} \otimes I \cdot \widehat{U}_{\mathrm{G}, \text { new }}=\widehat{U}_{\mathrm{E}, \mathrm{AGM}} \cdot \widehat{U}_{\mathrm{AGM}, \text { new }} \cdot
$$

Действия для решения Эрлера $\Phi_{\mathrm{E}}$, нового простого решения $\Phi_{\mathrm{G}}$ и кубического решения $\widehat{\Phi}_{\mathrm{AGM}}$ полевой теории струны Невё-Шварца (фермионной струны) с $\operatorname{GSO}(-)$-сектором были вычислены в работе [4], работах [12], [13] и работах [8], [9] соответственно и дали один и тот же результат:

$$
S\left[\Phi_{\mathrm{E}}\right]=S\left[\Phi_{\mathrm{G}}\right]=S\left[\widehat{\Phi}_{\mathrm{AGM}}\right]=\frac{1}{2 \pi^{2}} .
$$

\section{2. ОБОЗНАЧЕНИЯ}

Мы используем струнные поля $K, B, c, \gamma$ в полуструнных обозначениях [4], [8], [14]. Эти поля имеют БРСТ-преобразования

$$
\begin{aligned}
Q c & =c K c-\gamma^{2}, & Q \gamma & =c K \gamma-\frac{1}{2} \gamma K c-\frac{1}{2} \gamma c K, \\
Q \gamma^{2} & =c K \gamma^{2}-\gamma^{2} K c, & Q B & =K
\end{aligned}
$$


и удовлетворяют алгебраическим соотношениям

$$
\begin{array}{rlrlrl}
\{B, c\} & =1, & {[B, \gamma]} & =0, & {[c, \gamma]} & =0, \\
{[B, K]} & =0, & {[K, c]} & =\partial c, & {[K, \gamma]} & =\partial \gamma, \\
B^{2} & =c^{2}=0 . & &
\end{array}
$$

Далее нам понадобится оператор $(1+K)^{-1}$. Перепишем его, используя швингеровскую параметризацию [11]:

$$
\frac{1}{1+K}=\int_{0}^{\infty} d t e^{-t(1+K)}=\int_{0}^{\infty} d t e^{-t} \Omega^{t}
$$

\section{3. НОВЫЕ РЕШЕНИЯ}

В этом разделе мы представим новое решение уравнений движения

$$
Q \Phi_{+}+\Phi_{+} \star \Phi_{+}-\Phi_{-} \star \Phi_{-}=0, \quad Q \Phi_{-}+\Phi_{+} \star \Phi_{-}-\Phi_{-} \star \Phi_{+}=0
$$

фермионной ПТС. Простое аналитическое решение имеет вид $\left(\widehat{\Phi}_{\text {new }}=\Phi_{+}^{\text {new }} \otimes \sigma_{3}+\right.$ $\left.\Phi_{-}^{\text {new }} \otimes i \sigma_{2}\right)^{1)}$

$$
\begin{aligned}
\Phi_{+}^{\text {new }} & =\left(c(1+K) B c+2 B \gamma^{2}\right) \frac{1}{1+K} \\
\Phi_{-}^{\text {new }} & =\left(\gamma+c B K \gamma+\frac{1}{2} \gamma K B c+\frac{1}{2} \gamma B c K\right) \frac{1}{1+K}
\end{aligned}
$$

(далее мы опустим индекс "new" у полей $\Phi_{+}$и $\Phi_{-}$. Это решение является обобщением решения суперструнного уравнения движения [12]

$$
\Phi_{\mathrm{G}}=\left(c(1+K) B c+B \gamma^{2}\right) \frac{1}{1+K},
$$

которое отчасти (различие состоит в добавлении второго слагаемого, в бозонном решении присутствует только первое слагаемое) есть обобщение решения ЭрлераШнабла бозонного полевого уравнения движения [11].

Для описания калибровочной эквивалентности решений (3) и (4) целесообразно ввести функции [11]

$$
f=1, \quad g=\frac{1}{1+K}
$$

и переписать решения (3) и (4) в виде

$$
\begin{aligned}
& \Phi_{+}=f c \frac{K B}{1-f g} c g+f \gamma \frac{K B}{1-f g} \gamma g+f B \gamma(1-K) \gamma g, \\
& \Phi_{-}=f c \frac{K B}{1-f g} \gamma g+f \gamma \frac{K B}{1-f g} c g-\frac{1}{2} f \gamma K B c g+\frac{1}{2} f \gamma B c K g
\end{aligned}
$$

1) Мы также можем построить "приближенное" решение

$$
\Phi_{+}=\left(c+B \gamma^{2}\right)(1-K)+B \gamma^{2}, \quad \Phi_{-}=\gamma-\frac{1}{2} \gamma B c K+\frac{1}{2} \gamma K B c,
$$

которое формально удовлетворяет уравнениям движения (2). В случае чистого GSO $(+)$-сектора "приближенное" решение имеет вид $\Phi_{+}=\left(c+B \gamma^{2}\right)(1-K)$ [13]. 
и

$$
\Phi_{\mathrm{G}}=f c \frac{K B}{1-f g} c g+f B \gamma^{2} g .
$$

Если мы положим $f=g=F \equiv \Omega^{1 / 2}$, то получим решение АГМ и решение Эрлера.

\section{4. КАЛИБРОВОЧНАЯ ЭКВИВАЛЕНТНОСТЬ РЕШЕНИЙ}

4.1. Калибровочные преобразования $U_{\mathrm{E}, \mathrm{G}}$ и $\widehat{U}_{\mathrm{AGM}, \mathrm{new}}$. Следуя работе [11], мы можем построить калибровочное преобразование между решением (7) и решением Эрлера $\Phi_{\mathrm{E}}[12]$ :

$$
\Phi_{\mathrm{E}}=U_{\mathrm{E}, \mathrm{G}}^{-1}\left(\Phi_{\mathrm{G}}+Q\right) U_{\mathrm{E}, \mathrm{G}},
$$

где

$$
U_{\mathrm{E}, \mathrm{G}}=1-f B c g+M f^{\prime} B c g^{\prime}, \quad U_{\mathrm{E}, \mathrm{G}}^{-1}=1-f^{\prime} B c g^{\prime}+M^{-1} f B c g
$$

и функция $M$ определена следующим образом:

$$
M=\left(\frac{1-f g}{1-f^{\prime} g^{\prime}}\right) ;
$$

здесь $f^{\prime}=g^{\prime}=F$ и функции $f, g$ заданы формулами (5).

Калибровочное преобразование между решением (6) и решением АГМ имеет вид

$$
\widehat{\Phi}_{\mathrm{AGM}}=\widehat{U}_{\mathrm{AGM}, \mathrm{new}}^{-1}\left(\widehat{\Phi}_{\mathrm{new}}+\widehat{Q}\right) \widehat{U}_{\mathrm{AGM}, \text { new }},
$$

где

$$
\widehat{U}_{\mathrm{AGM}, \text { new }}=U_{+} \otimes I+U_{-} \otimes \sigma_{1}, \quad \widehat{U}_{\mathrm{AGM}, \text { new }}^{-1}=U_{+}^{-1} \otimes I+U_{-}^{-1} \otimes \sigma_{1}
$$

и

$$
\begin{aligned}
U_{+} & =1-f B c g+M f^{\prime} B c g^{\prime}, & U_{-} & =-f B \gamma g+M f^{\prime} B \gamma g^{\prime}, \\
U_{+}^{-1} & =1-f^{\prime} B c g^{\prime}+M^{-1} f B c g, & U_{-}^{-1} & =-f^{\prime} B \gamma g^{\prime}+M^{-1} f B \gamma g .
\end{aligned}
$$

Кроме того, мы положили $\widehat{Q}=Q \otimes \sigma_{3}, \widehat{\Phi}=\Phi_{+} \otimes \sigma_{3}+\Phi_{-} \otimes i \sigma_{2}$.

4.2. Калибровочное преобразование $\widehat{U}_{\mathrm{G}, \mathrm{new}}$. Построим калибровочное преобразование, связывающее два решения: решение уравнения движения в полевой теории суперструны и решение уравнений движения в полевой теории фермионной струны.

Рассмотрим калибровочное преобразование

$$
\widehat{\Phi}^{\prime}=\widehat{U}^{-1}(\widehat{\Phi}+\widehat{Q}) \widehat{U}
$$

и перепишем его в компонентах:

$$
\begin{aligned}
& \Phi_{+}^{\prime}=U_{+}^{-1}\left(\Phi_{+}+Q\right) U_{+}-U_{-}^{-1}\left(\Phi_{+}+Q\right) U_{-}+U_{+}^{-1} \Phi_{-} U_{-}-U_{-}^{-1} \Phi_{-} U_{+}, \\
& \Phi_{-}^{\prime}=U_{+}^{-1}\left(\Phi_{+}+Q\right) U_{-}-U_{-}^{-1}\left(\Phi_{+}+Q\right) U_{+}+U_{+}^{-1} \Phi_{-} U_{+}-U_{-}^{-1} \Phi_{-} U_{-} .
\end{aligned}
$$


Пусть $\Phi_{+}$и $\Phi_{-}$выбраны в виде (6) и

$$
U_{+}=U_{+}^{-1}=I, \quad U_{-}=-f B \gamma g, \quad U_{-}^{-1}=f B \gamma g .
$$

Тогда первое соотношение в (10) дает

$$
\begin{aligned}
U_{+}^{-1}\left(\Phi_{+}+Q\right) U_{+} & =f c \frac{K B}{1-f g} c g+f \gamma \frac{K B}{1-f g} \gamma g+f B \gamma(1-K) \gamma g \\
U_{-}^{-1}\left(\Phi_{+}+Q\right) U_{-} & =-f \gamma \frac{K B}{1-f g} f^{2} g^{2} \gamma g-f \gamma K B f g \gamma g \\
U_{+}^{-1} \Phi_{-} U_{-} & =-f \gamma \frac{K B}{1-f g} f g \gamma g \\
U_{-}^{-1} \Phi_{-} U_{+} & =f \gamma \frac{K B}{1-f g} f g \gamma g .
\end{aligned}
$$

Таким образом, мы получаем следующее выражение для $\Phi_{+}^{\prime}$ :

$$
\Phi_{+}^{\prime}=f c \frac{K B}{1-f g} c g+f B \gamma^{2} g
$$

Для второго соотношения в (10) имеем

$$
\begin{aligned}
U_{+}^{-1}\left(\Phi_{+}+Q\right) U_{-} & =-f c \frac{K B}{1-f g} f g \gamma g-f c K B \gamma g-\frac{1}{2} f \gamma K B c g-\frac{1}{2} f \gamma B c K g \\
U_{-}^{-1} \Phi_{+} U_{+} & =f \gamma \frac{K B}{1-f g} f g c g \\
U_{+}^{-1} \Phi_{-} U_{+} & =f c \frac{K B}{1-f g} \gamma g+f \gamma \frac{K B}{1-f g} c g-\frac{1}{2} f \gamma K B c g+\frac{1}{2} f \gamma B c K g, \\
U_{-}^{-1} \Phi_{-} U_{-} & =0
\end{aligned}
$$

следовательно, $\Phi_{-}^{\prime}=0$. Таким образом, мы получили калибровочную эквивалентность двух решений.

\section{5. ЗАКЛЮЧЕНИЕ}

Конструкция, представленная в настоящей статье, позволяет сделать вывод, что все решения, описывающие конечную точку конденсации тахиона, калибровочно-эквивалентны. В стандартной зигелевской калибровке тахион принадлежит GSO(-)сектору и нарушает суперсимметрию модели. Кроме того, можно ожидать, что аналитическое решение из работы [4], находящееся в чистом GSO(-)-секторе, нарушает суперсимметрию (в духе работы [15]). Чтобы проверить это утверждение, необходимо рассмотреть Р-сектор. В этом контексте было бы интересно проанализировать идею Кройтера построения ПТС Невё-Шварца-Рамона, где струнные поля несут произвольную картину и принадлежат большому гильбертовому пространству [16].

Благодарности. Работа частично поддержана РФФИ (грант № 08-01-00798), Программой поддержки ведущих научных школ (грант НШ-795.2008.1) и Федеральным агентством по науке и инновациям (гос. контракт 02.740.11.5057). 


\section{Список литературы}

[1] M. Schnabl, Adv. Theor. Math. Phys., 10:4 (2006), 433-501, arXiv: hep-th/0511286.

[2] Y. Okawa, JHEP, 04 (2006), 055, 36 pp., arXiv: hep-th/0603159.

[3] E. Witten, Nucl. Phys. B, 268:2 (1986), 253-294.

[4] T. Erler, JHEP, 01 (2008), 013, 15 pp., arXiv: 0707.4591.

[5] I. Y. Aref'eva, P. B. Medvedev, A. P. Zubarev, Nucl. Phys. B, 341:2 (1990), 464-498; Phys. Lett. B, 240:3-4 (1990), 356-362.

[6] C. R. Preitschopf, C. B. Thorn, S. A. Yost, Nucl. Phys. B, 337:2 (1990), 363-433.

[7] I. Y. Aref'eva, A. S. Koshelev, D. M. Belov, P. B. Medvedev, Nucl. Phys. B, 638:1-2 (2002), 3-20, arXiv: hep-th/0011117.

[8] И. Я. Арефьева, Р. В. Горбачев, П. Б. Медведев, ТМФ, 158:3 (2009), 378-390, arXiv: 0804.2017.

[9] I. Y. Aref'eva, R. V. Gorbachev, D. A. Grigoryev, P. N. Khromov, M. V. Maltsev, P. B. Medvedev, JHEP, 05 (2009), 050, 26 pp., arXiv: 0901.4533.

[10] E. Fuchs, M. Kroyter, JHEP, 10 (2008), 054, 25 pp., arXiv: 0805.4386; Analytical solutions of open string field theory, arXiv: 0807.4722.

[11] T. Erler, M. Schnabl, JHEP, 10 (2009), 066, 35 pp., arXiv: 0906.0979.

[12] Р. В. Горбачев, ТMФ, 162:1 (2010), 106-111.

[13] E. A. Arroyo, J. Phys. A, 43:44 (2010), 445503, 16 pp., arXiv: 1004.3030.

[14] T. Erler, JHEP, 05 (2007), 083, 23 pp., arXiv: hep-th/0611200; 084, 15 pp., arXiv: hep-th/0612050.

[15] I. Y. Aref'eva, P. B. Medvedev, A. P. Zubarev, Modern Phys. Lett. A, 6:11 (1991), 949-958.

[16] M. Kroyter, Superstring field theory in the democratic picture, arXiv: 0911.2962.

Поступила в редакцию 19.05.2010 